\title{
Méthodes de clôtures d'estuaires en rapport avec des problèmes de fondation et d'affouillement
}

\section{Foundation and scour problems \\ associated with various methods of closing an estuary}

\author{
PAR W. C. BISCHOFF VAN HEEMSIKERCK, \\ INGÉNIEYR EN CHEF DE LA SECTION D’ÉTUDES THÉOROUES ET EXPYRTMENTALES, \\ SERVICE DU DELTA, RHJSWATERSTAAT, LA HAYE
}

\begin{abstract}
Le sol des estauires hollandais est composé de matérianx à grains tres fins.

Ces matériaux supportent mal l'usure due aux courants d'eau qui sont à prévoir lors de la fermeture d'un bras de mer. Il y a donc lieu de craindre que des affoullements importants se produisent qui, à leur tour, provoqueraient des perturbations dans l'équilibre des couches sousjacentes.

Les inconvénients qui en résultent sont aggravés du fait que, selon toute vraisemblance, des paquets de sable mal tassé se rencontrent en cerfains endroits. Cette circonstance peut entrainer le danger qu'az cours de l'exécution des travaux, des pressions hydrauliques excessives se produisent dans la stratification de profondeur, ce qui risque de compromettre la résistance au cisaillement et d'accélérer de ce fait la perturbation de l'équilibre.

Sur la base de ces considérations, les avantages et inconvénients des différentes méthodes de fermeture constituent le sujet de cette communication.
\end{abstract}

\begin{abstract}
The beds of Dutch estuaries are made up of very fine materials, which are very vulnerable to wear by currents resulting from the closing of a sea inlet. Considerable scour may be expected to occur and, in turn, upset the equilibrium. of the deeper bed layers.

This unsatisfactory state of affairs is quite likely to be made worse by the occurrence of unstable local sand accumulations which, by causing excessively high water pressures in the deeper bed layers during the construction work, are liable to affect the shear strength of the materials and upset the equilibrium of the bed layers at an earlier stage.
\end{abstract}

This article discusses the merits and disadvantages of various estuary closure methods in the light of these factors.
La construction de barrages sur un certain nombre de bras de mer constitue une part importante du Projet Delta. Dans tous les endroits qui sont indiqués sur la figure 1 (voir carte), des barrages seront construits.

En principe, on peut évidemment adopter diverses méthodes de travail pour construire ces barrages.

En premier lieu, les digues peuvent avancer en partant des rivages. On l'a indiqué schéma- tiquement sur la figure 2 . On peut y voir que l'orifice par lequel la marée peut pénétrer dans l'estuaire devient plus étroit à mesure que les travaux progressent.

Par suite de ce rétrécissement, les niveaux de la nappe du côté inférieur de la digue suivront avec un retard qui ira en croissant le rythme de la marée qui se produit à l'extérieur de la digue. La dénivellation à l'orifice qui reste augmentera à mesure que cet orifice se rétrécira, 


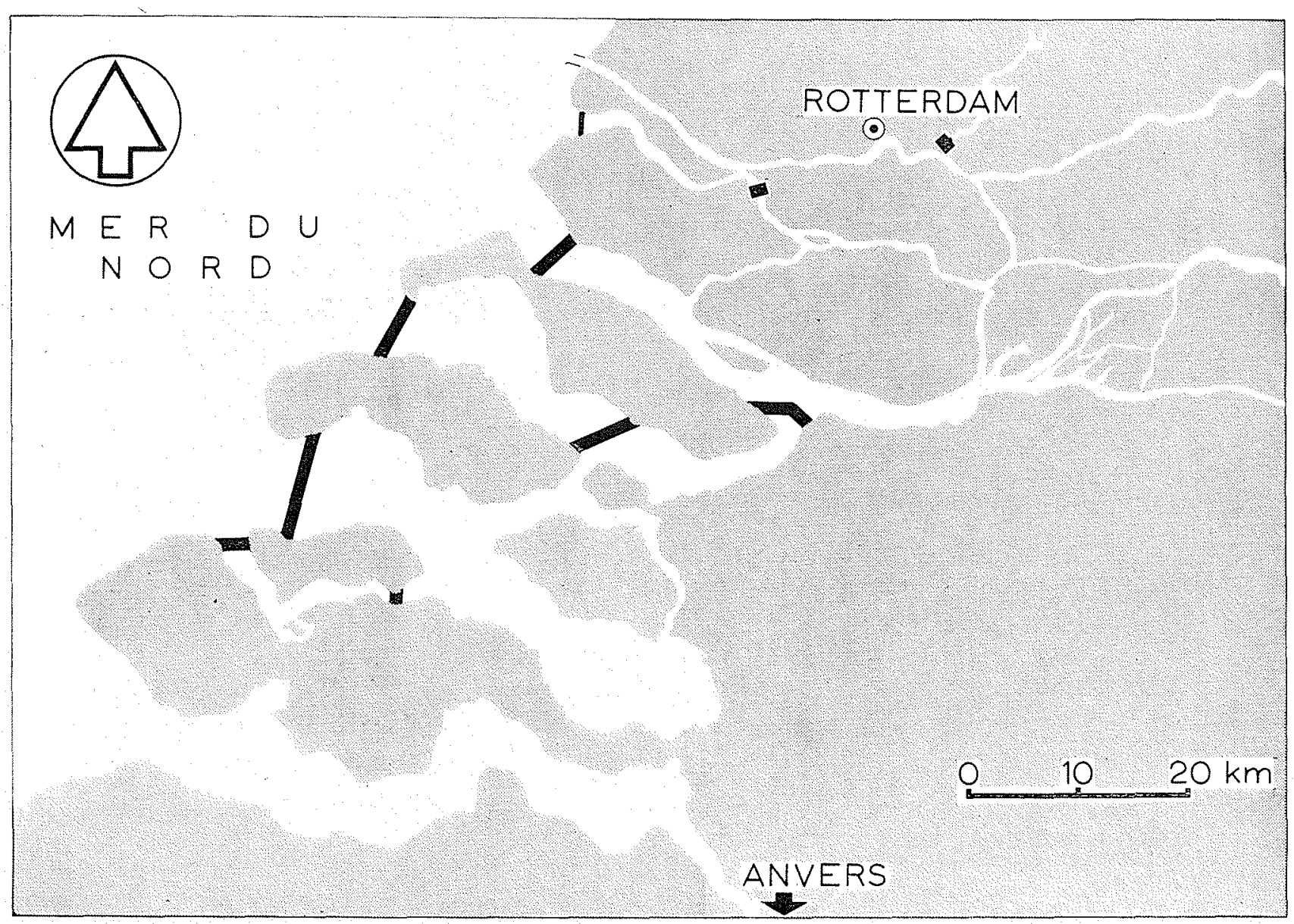

FIG. 1

A une dénivellation croissante, correspondra une augmentation des vitesses du courant. Pour les grands bras de mer néerlandais, cela peut se voir sur la figure 3 . Les vitesses de courant que l'on prévoit sont portées en fonction de l'ouverture de la passe.

Ce graphique montre qu'en suivant cette méthode on peut prévoir, lors de marées moyennes en mer, des vitesses de courant qui atteignent 5 à $6 \mathrm{~m} / \mathrm{s}$ dans la dernière phase de la fermeture.

Il est évident que de tels courants à si grandes vitesses, soulèvent déjà des problèmes qui leur sont propres, surtout lorsqu'on a affaire à un fond affouillable comme c'est le cas aux Pays-Bas.

A cela s'ajoute encore qu'à cause du rétrécissement horizontal de la passe, il faut prévoir une forte contraction, ainsi que des chapelets de tourbillons intenses près des têtes de digues (fig. 4). Les chapelets de tourbillons, du point de vue des affouillements, constituent un danger si grave que cette méthode de fermeture n'est envisagée que s'il y a un fond résistant (de la roche) ou que si par suite d'une faible amplitude, on s'attend à de faibles courants. Encore

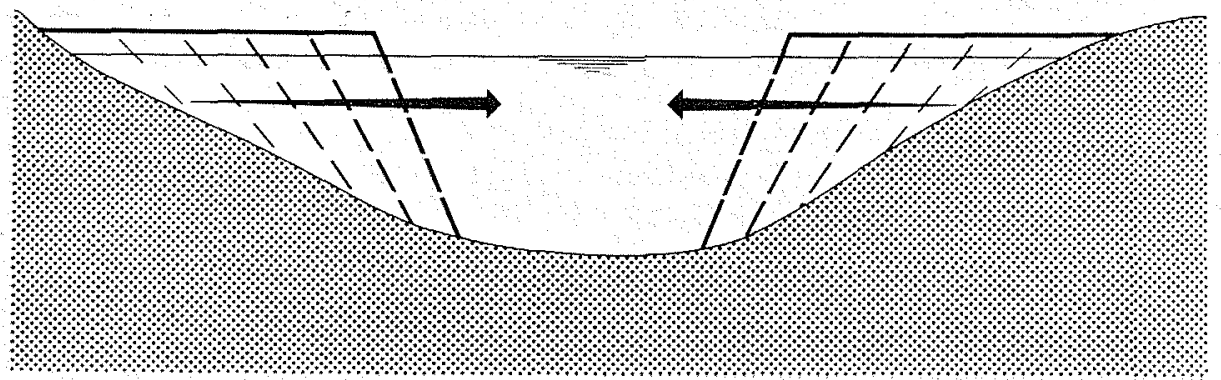

FIG. 2

Fermeture par assemblage en partant des denx rives, 


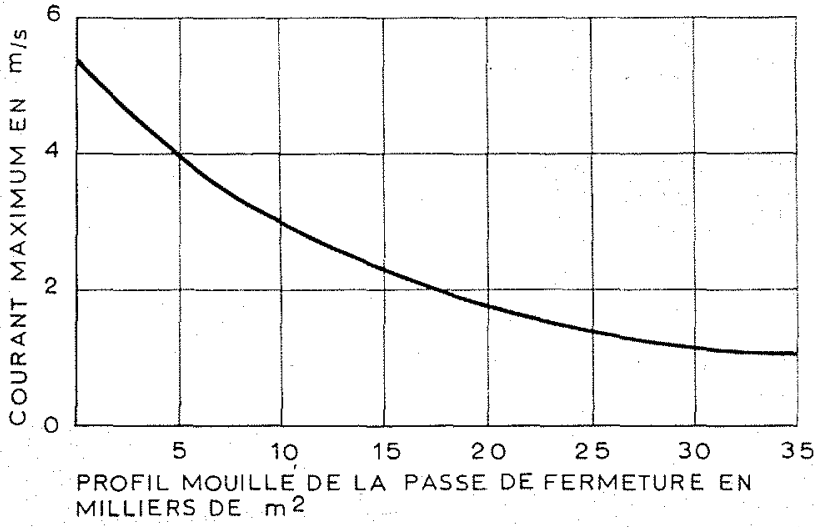

FIG. 3

Fermeture du barrage de I'estuaire de Brouwershaven.

\section{DIRECTION DE L'ÉCOULEMENT}

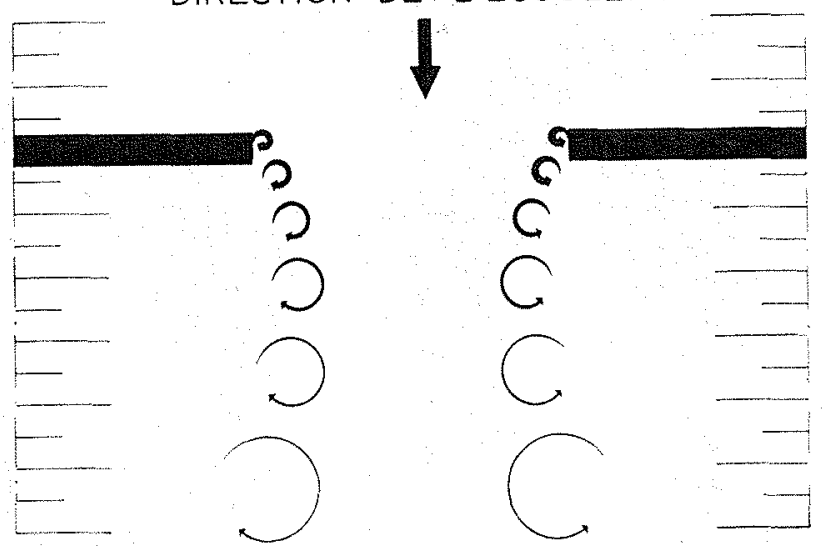

FIG. 4

Chapelets de tourbilions lors de la fermeture par remblayage en partant des deux rives.

FIG. 5

Grevelingen dam.

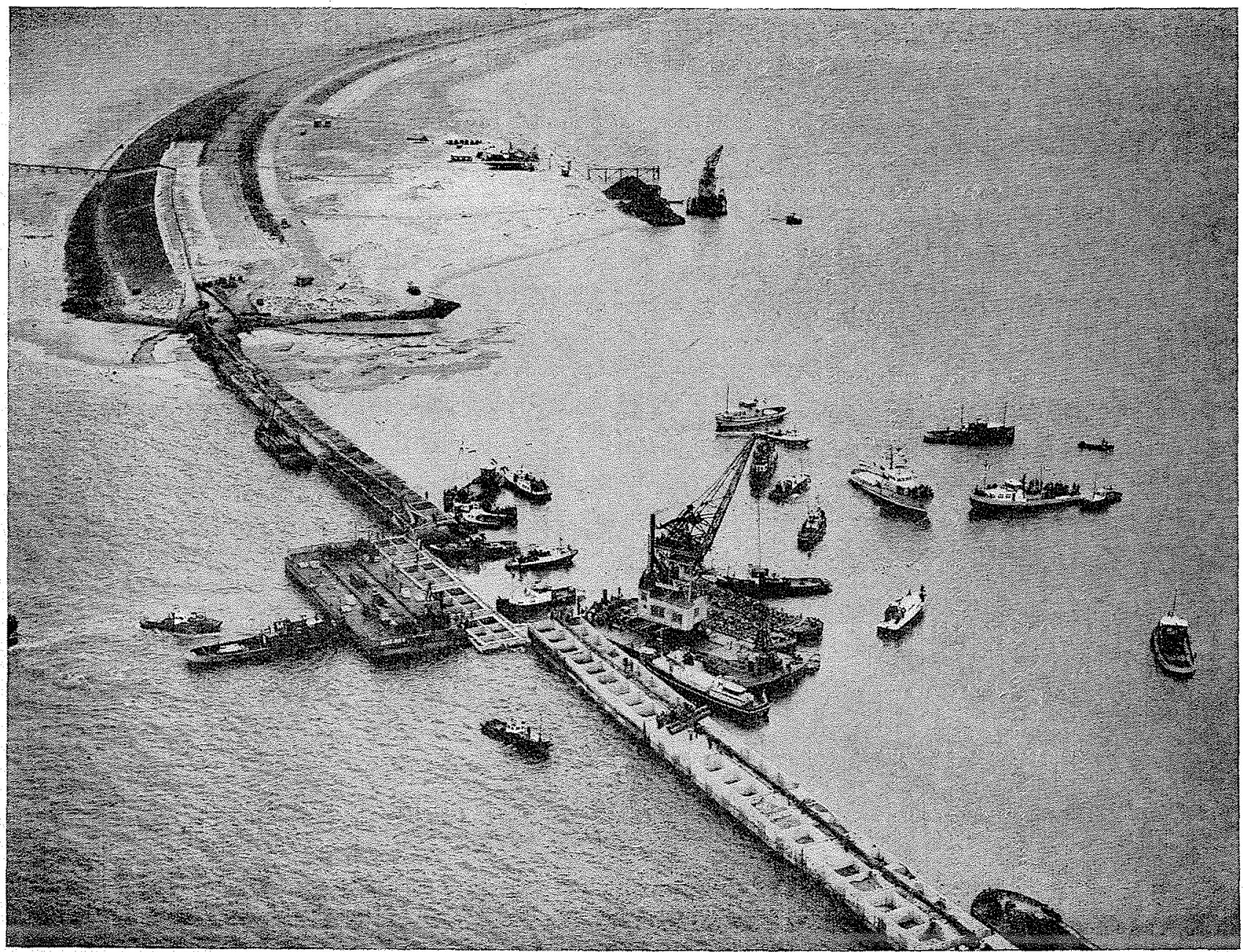




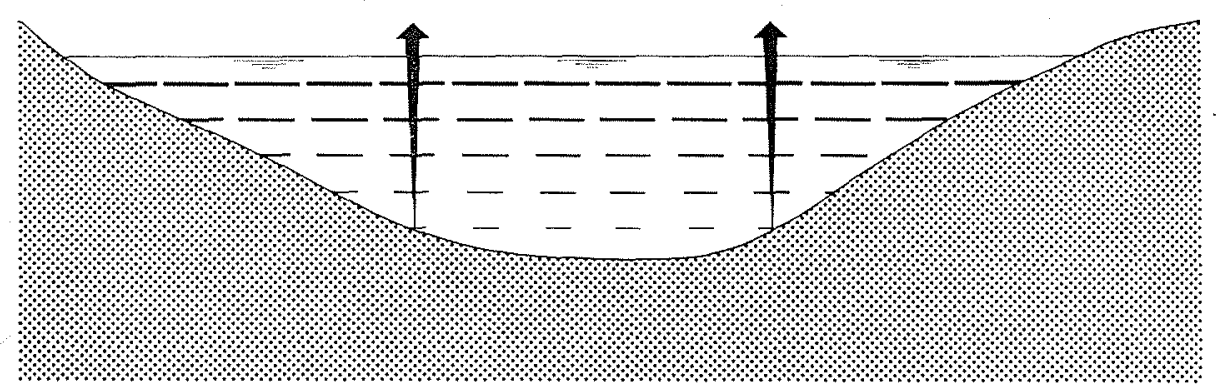

FIG. 6

Fermeture par remblayage en partant du fond.

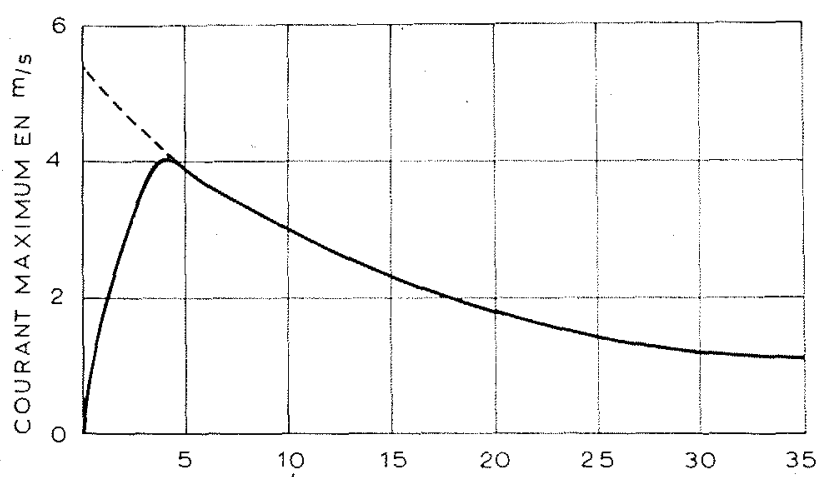

PROFIL MOUILLÉ DE LA PASSE DEFERMETURE EN MILLIERS DE $\mathrm{m}^{2}$

Fis. 7 Fermeture par exhaussement. Estuaire de Brouwershaven.

essaiera-t-on le plus souvent d'échapper aux moments les plus défavorables en procédant à la fermeture réelle pendant un court laps de temps, savoir pendant les mortes eaux. Afin d'atteindre l'allure qui s'impose dans le travail, on utilise le plus souvent aux Pays-Bas des caissons. Quand la mer est étalée, on les met en place sur un seuil qui a été préparé d’avance.
C'est de cette manière qu'on a fermé cette année-ci la passe qu'il y avait dans le canal de Grevelingen. Comme la marée y monte de deux côtés à la fois, dans la dernière phase de la fermeture, les courants ne dépassaient pas une vitesse de $2,5 \mathrm{~m} / \mathrm{s}$. Aussi cette fermeture put-elle se réaliser à vive allure à l'aide de caissons.

La figure 5 illustre les travaux dans une phase avancée.

Comme je l'ai dit, cette méthode ne convient pas pour fermer nos larges bras de mer. Leur fermeture, même à une allure aussi vive que possible, prendrait pas mal de temps. Les grandes vitesses de courant et les chapelets de tourbillons intenses auraient alors assez de temps pour accomplir leur travail destructeur.

Une seconde méthode consiste à construire le barrage par couches horizontales déposées en même temps sur toute la largeur (voir la figure 6). Avec cette méthode aussi, l'orifice est progressivement comblé, et ici encore ce bouchage a comme conséquence d'accroître la dénivellation et les vitesses de courant dans la passe.

Ceci se voit sur la figure 7 où les vitesses de courant que l'on prévoit sont également portées

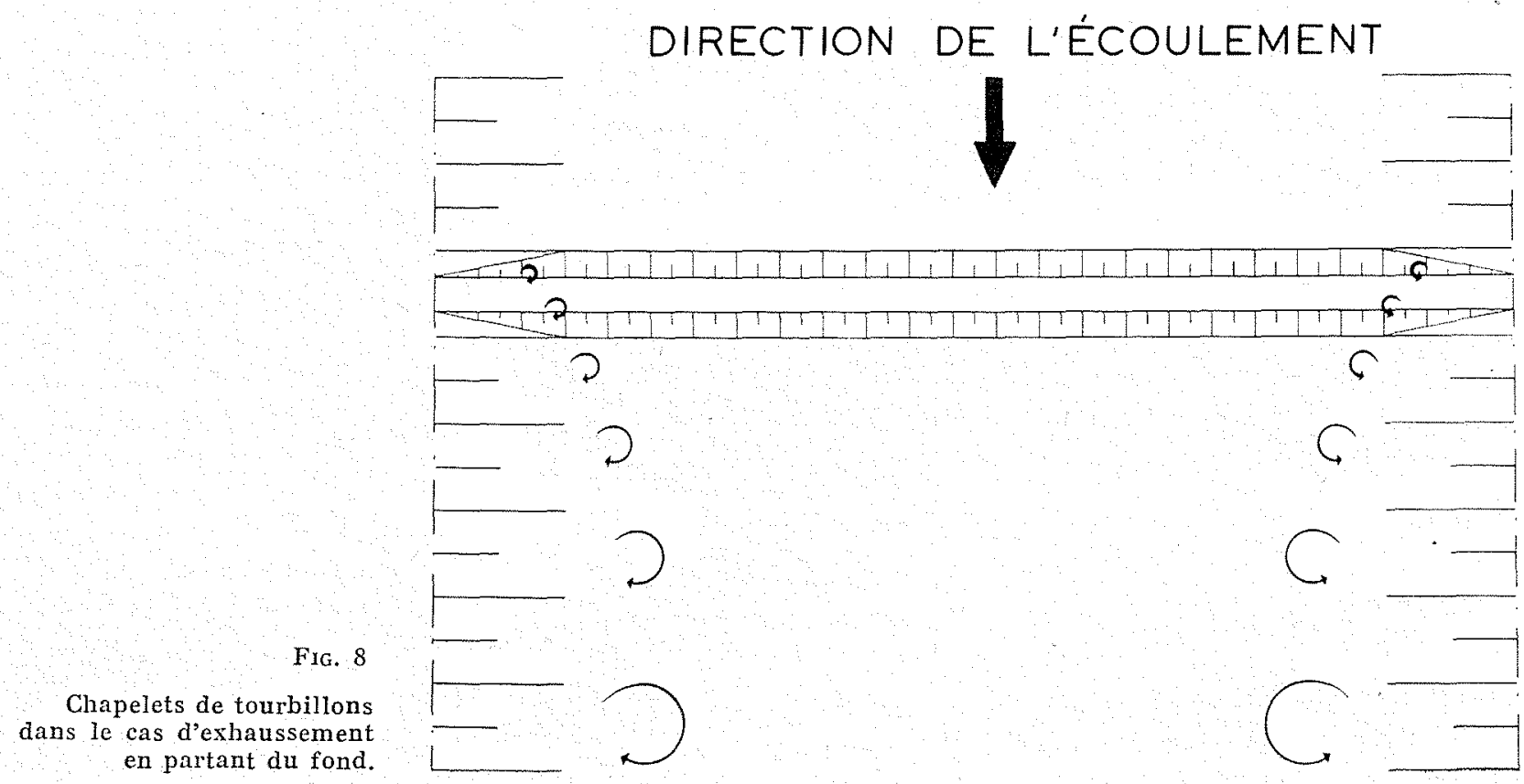




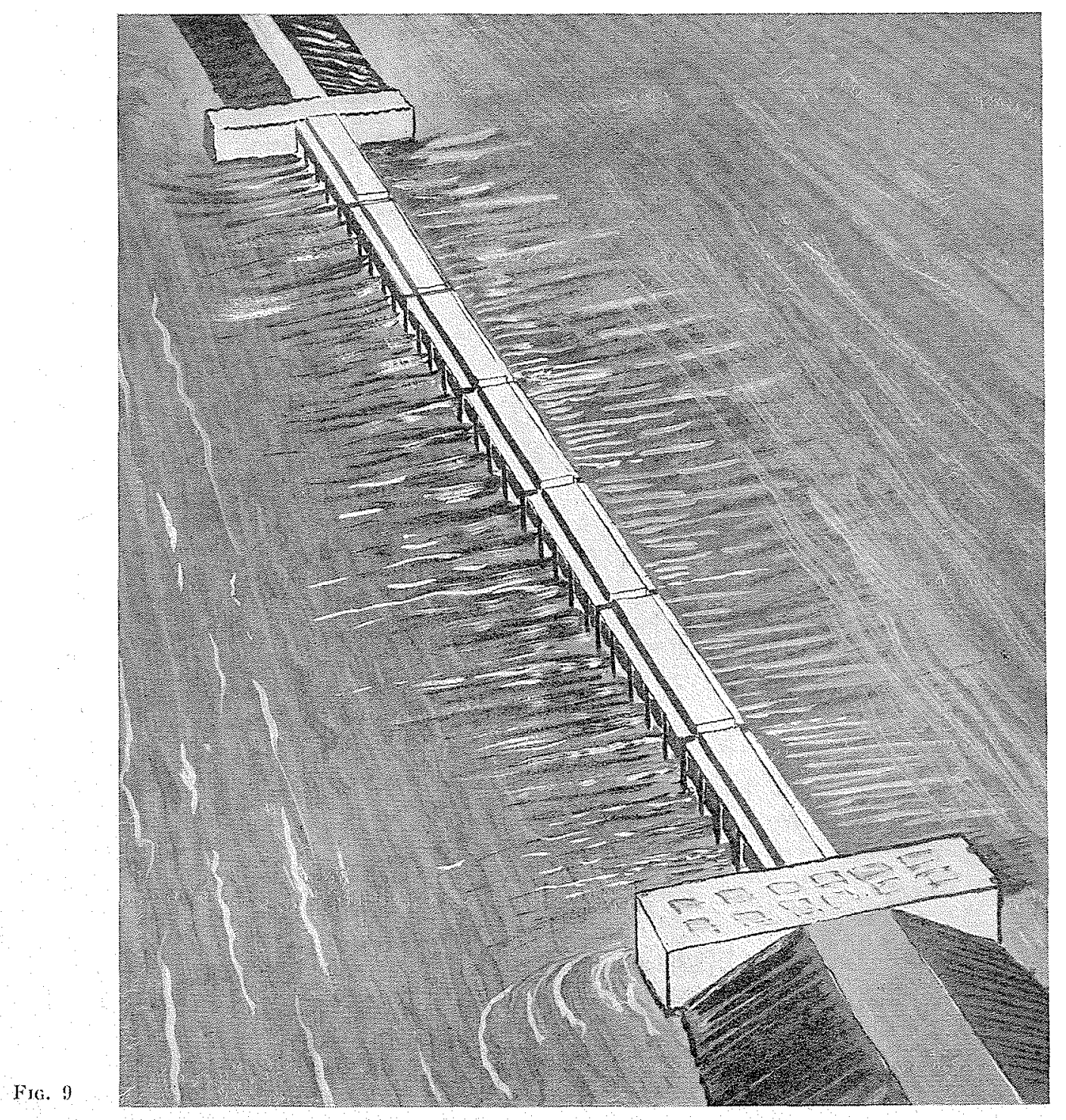

en fonction de l'ouverture. A titre de comparaison, on a encore tracé sur ce graphique, en la répétant en pointillé, la courbe qui représente la fermeture en sens horizontal. Il en résulte qu'il y a à cet égard une différence entre les deux méthodes. Lors d'un rétrécissement horizontal, les vitesses de courant continuent à crồtre jusqu'à ce que la fermeture soit tout à fait terminée. Par contre, en procédant à la construction de la digue par couches horizontales, on obtient à un certain moment un déversoir à nappe libre. A partir de ce moment-là, les vitesses de courant, non seulement n'augmentent plus lors de l'élévation du seuil, mais encore diminuent.

Cela n'empêche toutefois pas que, pour fermer les grands bras de mer néerlandais en utilisant cette méthode, il faudrait encore tenir compte de vitesses qui atteignent environ $4 \mathrm{~m} / \mathrm{s}$.

En appliquant cette méthode, des chapelets de tourbillons se produiront encore le long des bords de la passe. Bien que ces tourbillons en chapelets soient moins intenses que ceux que j'ai indiqués pour une fermeture en sens horizontal, ils peuvent cependant causer, avec les vitesses de courant indiquées, des affouillements en aval de la passe.

Cette méthode ouvre en outre la possibilité que la nappe se jette le long du talus d'aval en bas. On parlera dans un des autres exposés, de cette nappe, ainsi que d'ailleurs des chapelets de tourbillons.

Bien que la construction graduelle de digues par couches successives présente plus de possibilités qu'un rétrécissement horizontal de la passe, des dangers restent liés à la fermeture graduelle d'un bras de mer.

C'est pourquoi on envisage également une fermeture instantanée pour obturer les grands bras de mer. Elle pourrait se réaliser à l'aide de cais- 


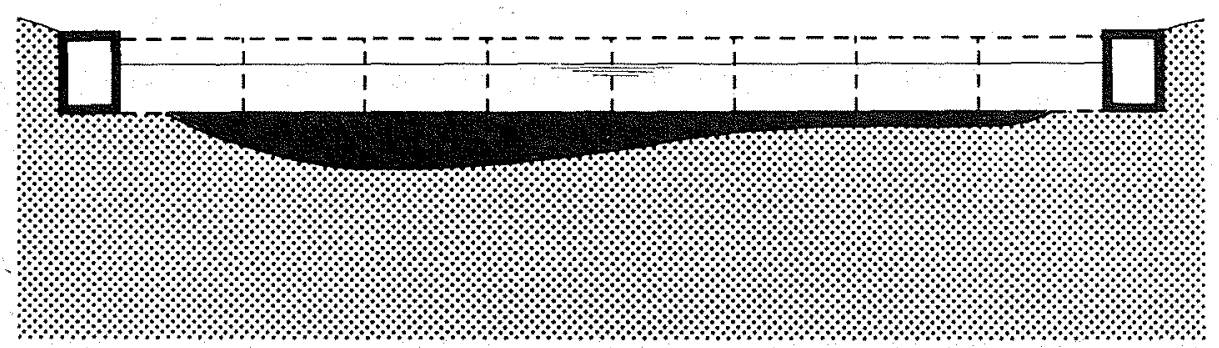

Frg. 10

Fermeture au moyen de caissons at pertuis.

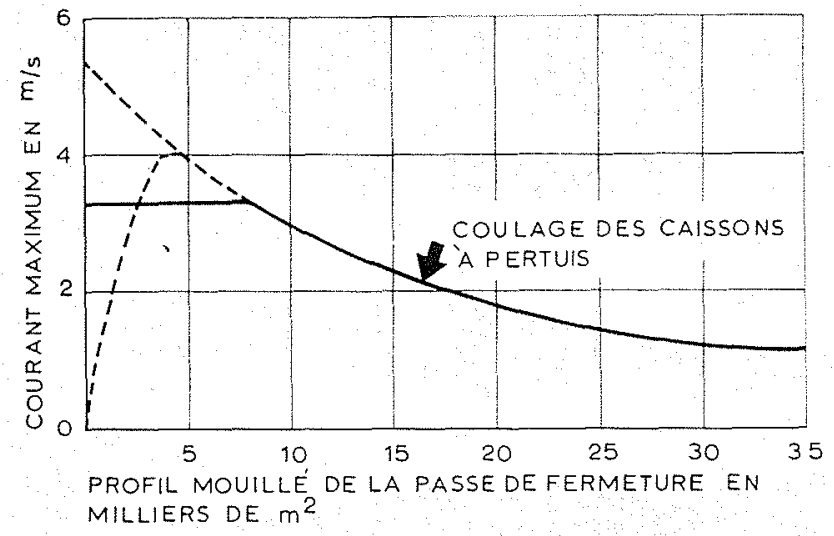

FIG. 11 sons à pertuis. Les caissons étant évidés, le mouvement de la marée peut encore avoir lieu librement, même lorsque tous les caissons ont été mis en place. A mer étale, on abaisse alors simultanément les vannes qui se trouvent dans les caissons de façon à obturer entièrement les pertuis.

Les caissons à pertuis ont toutefois une hauteur limitée et ils demandent un seuil plat pour y être posés. Cela signifie dans la pratique qu'is sont coulés sur un seuil qui a déjà été préparé dans l'année qui précède la fermeture. Ce seuil constitue un rétrécissement du profil d'écoulement de la passe qui, surtout en cas de fermeture de chenaux profonds, peut encore donner

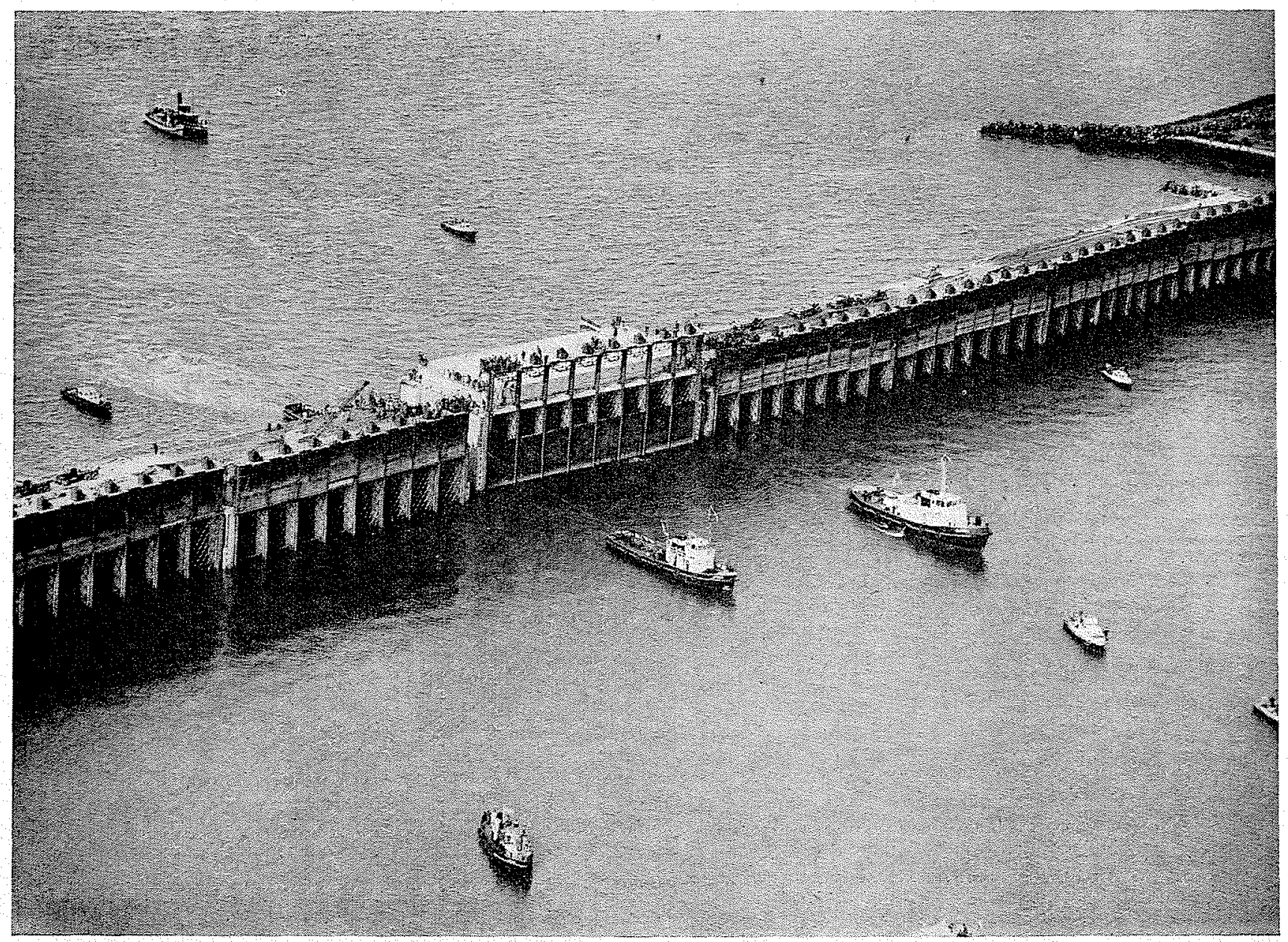

Fig. 12 

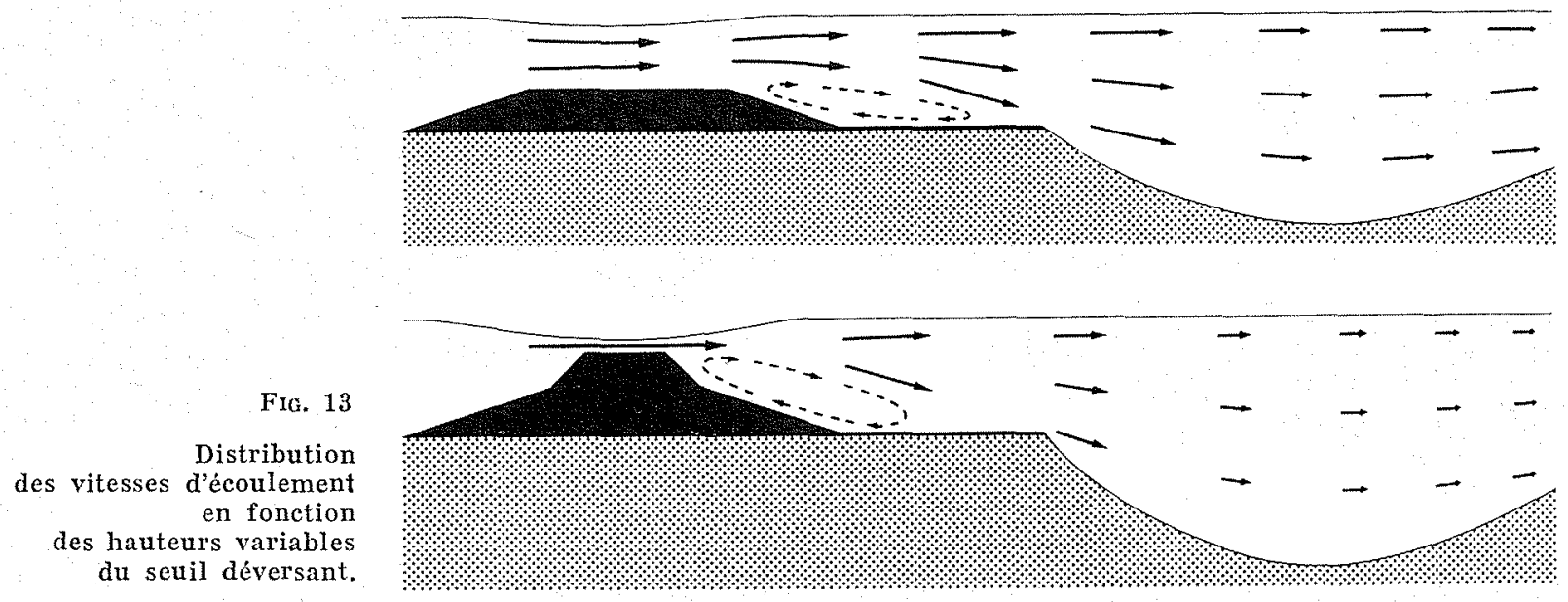

lieu à de grandes vitesses de courant. En outre, les caissons ne sont évidemment pas entièrement «évidés» et les éléments de construction offrent une certaine résistance à l'eau qui passe.

La méthode revient donc en pratique à un compromis entre une fermeture graduelle et une fermeture instantanée.

Quand on construit le seuil, on commence pour ainsi dire une fermeture graduelle. Les vitesses de courant augmentent selon la courbe que j’ai déjà montrée (fig. 11).

Dès que le senil est assez haut, les caissons sont coulés. Par suite de la résistance des caissons, les vitesses augmentent encore un peu. On empêche toutefois que les vitesses augmentent davantage en abaissant simultanément, à mex étale, les vannes des caissons qui sont déjà en place.

Les plus grandes vitesses de courant qui se produiront finalement sont déterminées par la hauteur de construction des caissons et par la profondeur du chenal. En général, la méthode perd de son intérêt à mesure que la profondeur du chenal augmente.

En 1961, le canal du Veerse Gat, qui avait une profondeur de 20 mètres, a été fermé à l'aide de caissons évidés.

Comme on peut le constater sur la figure 12 , le principe a eu un plein succès à cet endroit; aussi cette fermeture a-t-elle eu lieu sans diffcultés.

En énumérant les avantages et les désavantages, j'ai toujours mis l'accent jusqu'ici sur les vitesses du courant. J'ai supposé tacitement qu'elles constitueraient le facteur direct pour mesurer les affouillements.

Une simple étude de la figure 13 apprend toutefois que cela n'est pas tout à fait justifié. Cette figure montre qu'outre la vitesse du courant interviennent aussi le débit et la hauteur du seuil.

La partie supérieure de cette figure représente le courant qui se produit lorsque le seuil n'est pas encore très élevé. Au-dessus du seuil, il y a une certaine vitesse et une certaine profondeur d'eau. Ces facteurs déterminent ensemble le débit qui passe sur le seuil. Ce débit se répand derrière le seuil sur la profondeur disponible et finit, dans la figure, à droite, par une certaine vitesse du courant.

La partie inférieure de la figure représente le courant qui se produit lorsque le seuil est plus élevé.

La vitesse au-dessus du seuil est, dans ce caslà, bien plus grande, mais la profondeur de l'eau au-dessus du déversoir est plus petite.

Il est done possible que, malgré la plus grande vitesse du courant, un débit plus réduit passe dans ce cas-ci par-dessus le seuil. Si tel est le cas, ce débit plus réduit se répandra sur la même profondeur que dans le premier cas, de sorte que les vitesses du courant aux limites de la protection de fond seront finalement plus petites que dans le cas d'un seuil plus bas.

Il y a évidemment d'autres facteurs qui jouent un rôle, comme par exemple, la turbulence. L'explication que je viens de donner est donc trop simple. Elle rend toutefois compréhensible que des vitesses plus grandes au-dessus du seuil n'entraînent pas forcément des affouillements plus grands si le débit est plus petit.

Que ce débit diminue, en effet, lors de la fermeture des bras de mer néerlandais, cela se voit sur la figure 14, ou en plus des vitesses maximales qu'on a déjà vues, le débit figure maintenant aussi en fonction de l'ouverture de la passe.

Il en résulte que le débit diminue en effet, tandis que la vitesse augmente. On ne peut donc pas prevoir a priori quels sont les affouillements.

On a évidemment fait des expérimentations.

La figure 15 en donne le résultat schématiquement.

Sur cette figure, nous avons porté $\cdots \cdots$ fon- 


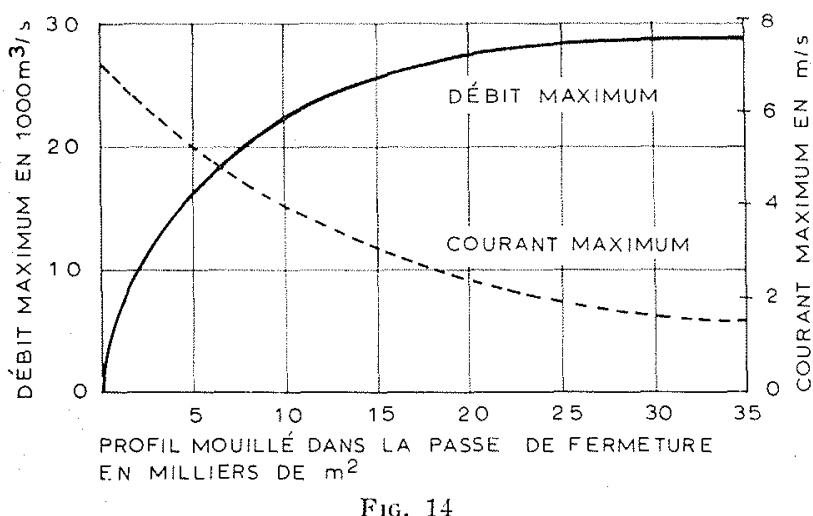

Fermeture de l'estuaire de Brouwershaven.

deurs d'affouillements qui se sont produites en fonction des rapports des hauteurs du seuil aux profondeurs du chenal. Ces essais ont èté effectués pendant une série de périodes d'écoulement de diverses durées.

La figure indique également, en pointillé et grosso modo, le déroulement des vitesses de courant correspondantes.

Dans les conditions valables pour les PaysBas, l'affouillement semble augmenter lorsque la hauteur du déversoir croît jusqu'à une valeur d'environ les trois quarts de la profondeur d'eau. Lorsque la hauteur du déversoir est plus grande, la diminution du débit prévaut et le danger d'affouillement diminue graduellement. Les plus grands affouillements ne correspondent pas exactement aux plus grandes vitesses du courant, ce qui peut constituer un facteur important à envisager, bien que la différence semble peutêtre modeste sur cette figure.

Lors de la fermeture du Veerse Gat à l'aide de caissons, dont on a vu une photo, la profondeur de l'eau éta: ${ }^{\prime}$........ nn - ètres et la hauteur

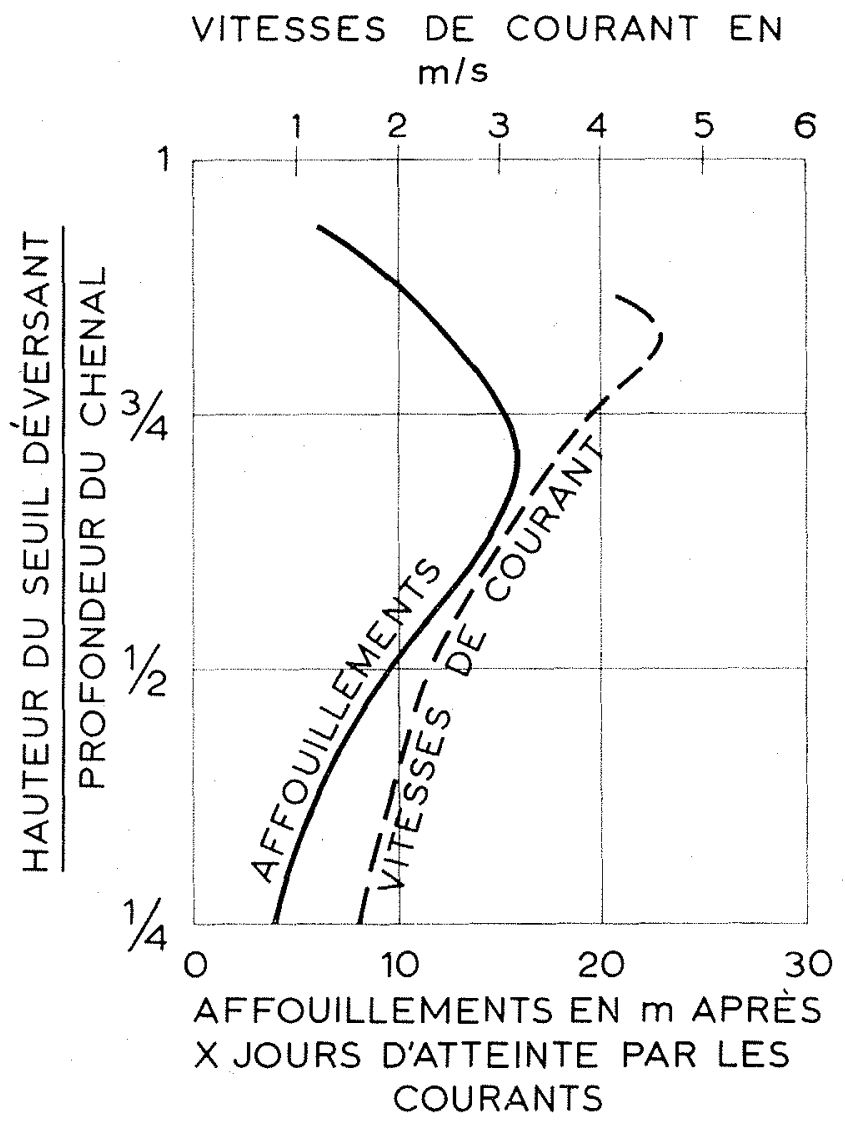

FIG. 15

du seuil d'environ 10 mètres. Le rapport entre ces deux grandeurs était done $1 / 2$. A ce momentlà, on n'avait donc pas atteint, de loin, la plus grande attaque du courant. En fermant l'ouverture qui restait à l'aide de vannes, toutes à la fois, on a pu éviter entièrement cette attaque maximale du courant.

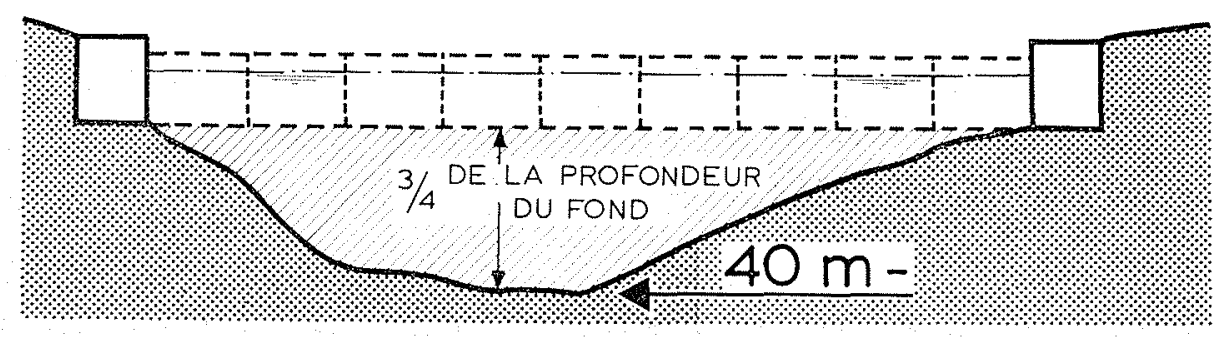

FIG. 16

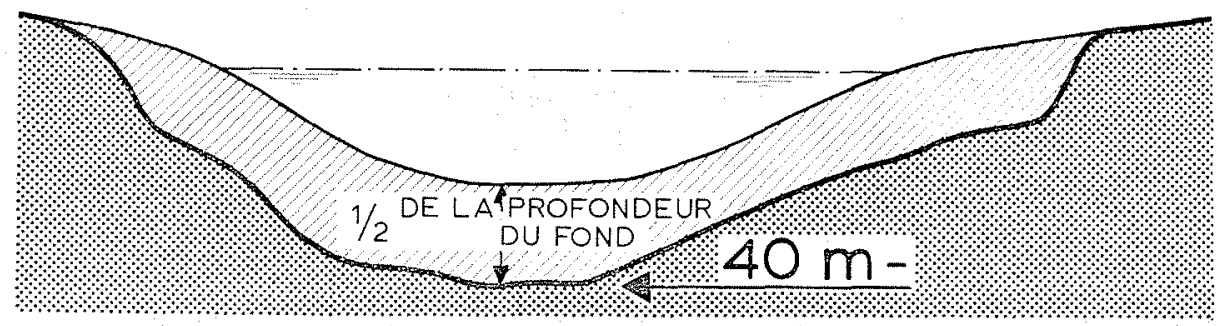




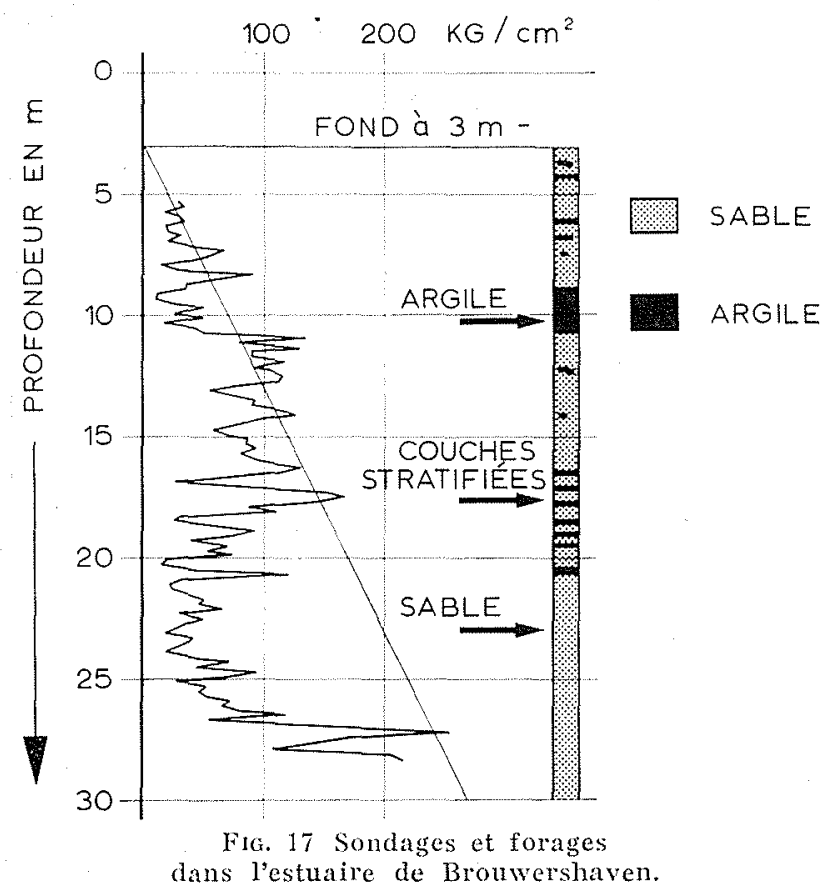

Si le chenal avait été beaucoup plus profond (par exemple $40 \mathrm{~m}$ ), il aurait fallu, pour la même hauteur des caissons, un seuil d'une hauteur qui fût exactement les $3 / 4$ de la profondeur de l'eau. Pendant une durée prolongée, l'attaque maximale du courant se serait produite, et il en serait résulté des affouillement profonds.

En remblayant graduellement le seuil, on aurait rencontré moins de difficultés. On n'est plus lié à une hauteur de caisson limitée, ni à une surface horizontale de la partie supérieure du seuil.

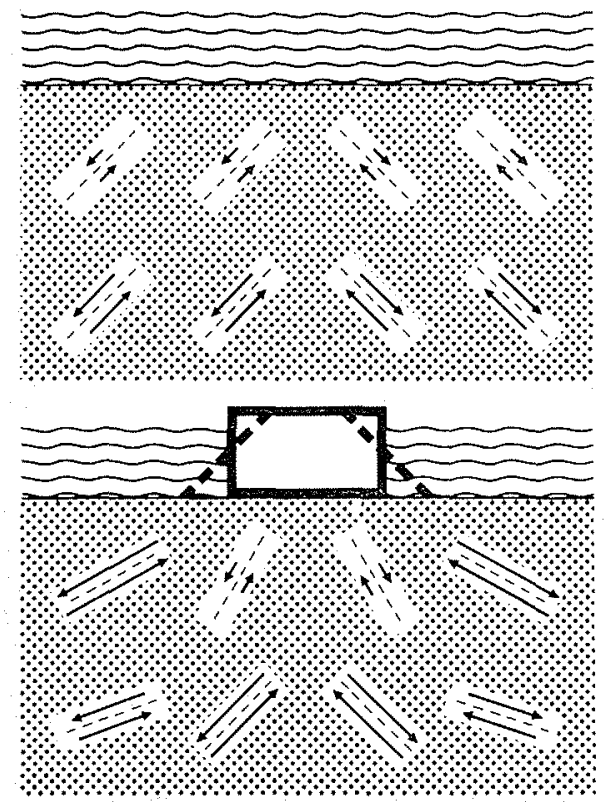

En hiver surtout, le seuil peut être adapté à la profondeur du chenal, et l'on peut ainsi éviter, pendant une durée plus longue, une vive attaque du courant (fig. 16).

On peut être tenté de conclure, en s'appuyant sur ces considérations, qu'une construction graduelle du seuil bien étudiée offre plus d'avantages pour des chenaux profonds qu'une fermeture avec des caissons évidés, mais que la fermeture avec des caissons est à préférer pour barrer des chenaux de profondeur moindre.

Cette conclusion non plus ne vaut pas dans toutes les circonstances, en particulier pas pour les bras de mer néerlandais, où il y a du sable mal tassé.

La figure 17 montre le résultat d'un forage et d'un sondage qui ont été effectués dans un des bras de mer néerlandais. En faisant un sondage dans un massif de sable homogène, on doit s'attendre à ce que la résistance de sondage croisse à peu près à mesure que la profondeur augmente. La résistance de sondage approximativement théorique à laquelle on doit s'attendre pour un angle de friction (o) de $30^{\circ}$ est indiquée sur le graphique. Cependant la résistance de friction qui a été mesurée se révèle, sur un large intervalle, bien inférieure à la résistance théorique.

Là où il y a des couches d'argile, cela est normal. Sur la partie inférieure du graphique, où il y a du sable pur d'après le déponillement de l'échantillon, la résistance de sondage ne s'élève pourtant pas jusqu'à la courbe théorique qu'on prévoyait. La cause de ce fait n'est point connue avec certitude; provisoirement nous pensons que cette résistance de sondage trop faible qui se produit dans le sable est provoquée par le tassement lâche de ce sable.

On voit cependant qu'il y a bien toujours une certaine résistance de sondage, si bien que nous devons nous demander quelle signification il faut lui reconnaître.

Pour pouvoir répondre à cette question, nous devons en premier lieu nous représenter ce qui se passe dans le sol en profondeur. C'est indiqué sur la figure 18, à titre d'exemple. Cette figure fait voir comment, lors du remblayage d'un seuil ou lors de la mise en place d'un caisson, les fatigues de cisaillement, dans le sol en profondeur,

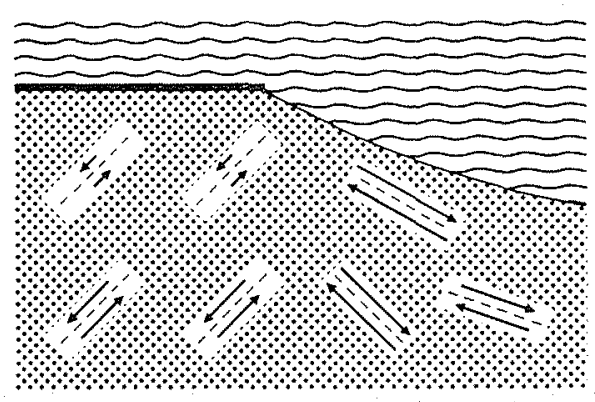

FIG. 18

Variations

des fatigues de cisaillement dans les profondeurs du terrain lors de l'exécution des travaux. 
FATIGUES DE CISAILLEMENT

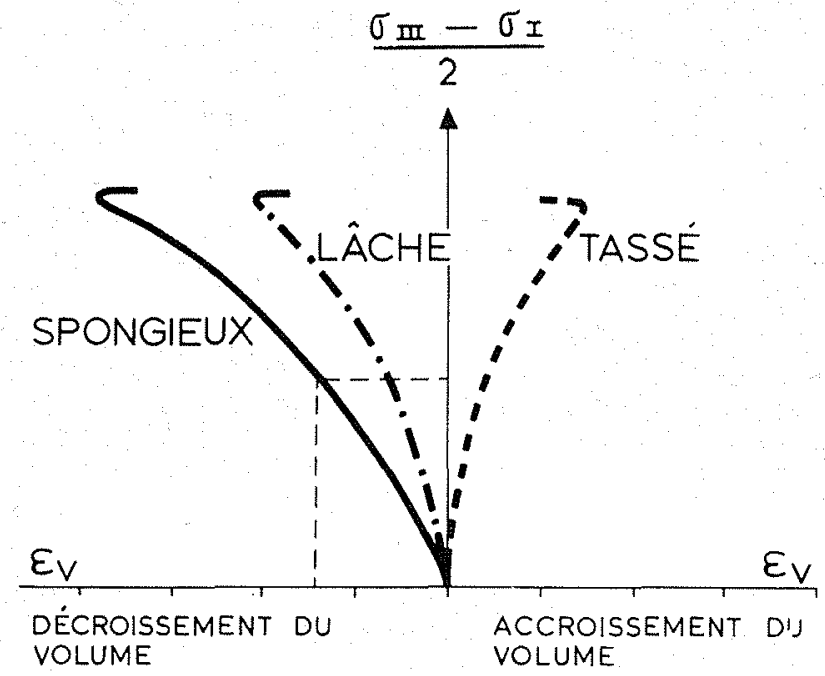

Fig. 19 Propriétés de métamorphose du sable.

changent de grandeur et de direction. La partie supérieure de la figure indique les fatignes de cisaillement avant la construction du seuil; la partie inférieure les indique après la construction.

Un affouillement ne provoque pas le chargement, mais le déchargement du sol en profondeur, et il est évident qu'en conséquence, les fatigues de cisaillement dans le sol en profondeur changeront.

Il est naturellement possible de donner encore d'autres exemples, mais voici la conclusion générale.

Par suite de l'exécution des travaux, l'aspect des fatigues de cisaillement changera dans le sol en profondeur, pour plusieurs raisons.

Le sable sec peut en général résister facilement à de tels changements des fatigues de cisaillement. Il subit néanmoins un changement de volume. A ce sujet, la figure 19 indique schématiquement la relation qu'il y a entre la fatigue de cisaillement et le changement de volume.

Dans les sables bien tassés, il se produira une augmentation de volume; dans les sables à tassement lâche, il y aura une diminution de volume. Dans ce dernier cas, la diminution de volume qui est nécessaire pour neutraliser une certaine fatigue de cisaillement sera d'autant plus grande que le sable a un tassement plus lâche.

Si les espaces intersticiels sont pleins d'eau, ce caractère subsiste. Les fatigues de cisaillement qui se produisent par l'apport d'une charge ou à la suite d'un affouillement doivent être neutralisées par le squelette de grains, mais cela se fait, comme la figure 19 le montre, avec des changements de volume.

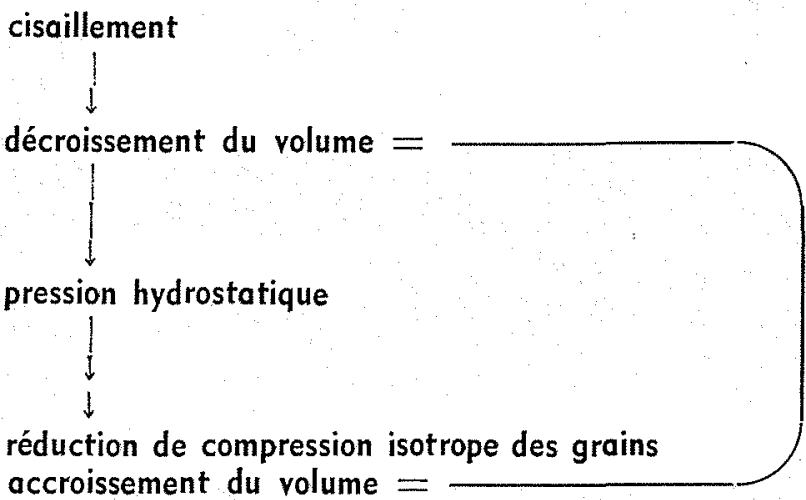

FIG. 20

Puisque l'eau n'est qu'en très faible mesure compressible, cela n'est donc pas possible, sans qu'un excès de pression hydrostatique ne se produise.

Par conséquent, le squelette de grains sera décomprimé dans tous les sens et il augmentera donc de nouveau de volume.

En résumé, la marche des idées s'établit comme suit (fig. 20).

Au cours de l'exécution des travaux, il se produit des fatigues de cisaillement. Elles ne peuvent être neutralisées, dans du sable à tassement lâche, que si une décroissance de volume survient. Il en résultera une surpression hydrostatique; le squelette de grains en éprouvera une décharge. Ceci provoque une augmentation de volume qui, déduction faite de la compression de l'eau, doit être égale à la diminution de volume qui a déjà été signalée.

Dans du sable bien tassé, c'est naturellement le contraire. Là, quand il y aura un changement dans les fatigues de cisaillement, il se produira des sous-pressions hydrostatiques.

Quand on ajoute lentement les charges, les pressions hydrostatiques supplémentaires restent faibles, car l'eau a le temps de fuir, ce qui a comme résultat de compenser l'excès de pression.

Il y a toutefois aussi des chargements instantanés, comme ceux que produit le «coulage» d'un caisson, l'attaque du flot et la poussée du vent sur ce caisson coulé.

L'affouillement lui-mème s'effectue bien lentement, mais dans les talus qui en résultent, des glissements peuvent se produire et ils entraînent bien un déchargement rapide du sol en profondeur. 


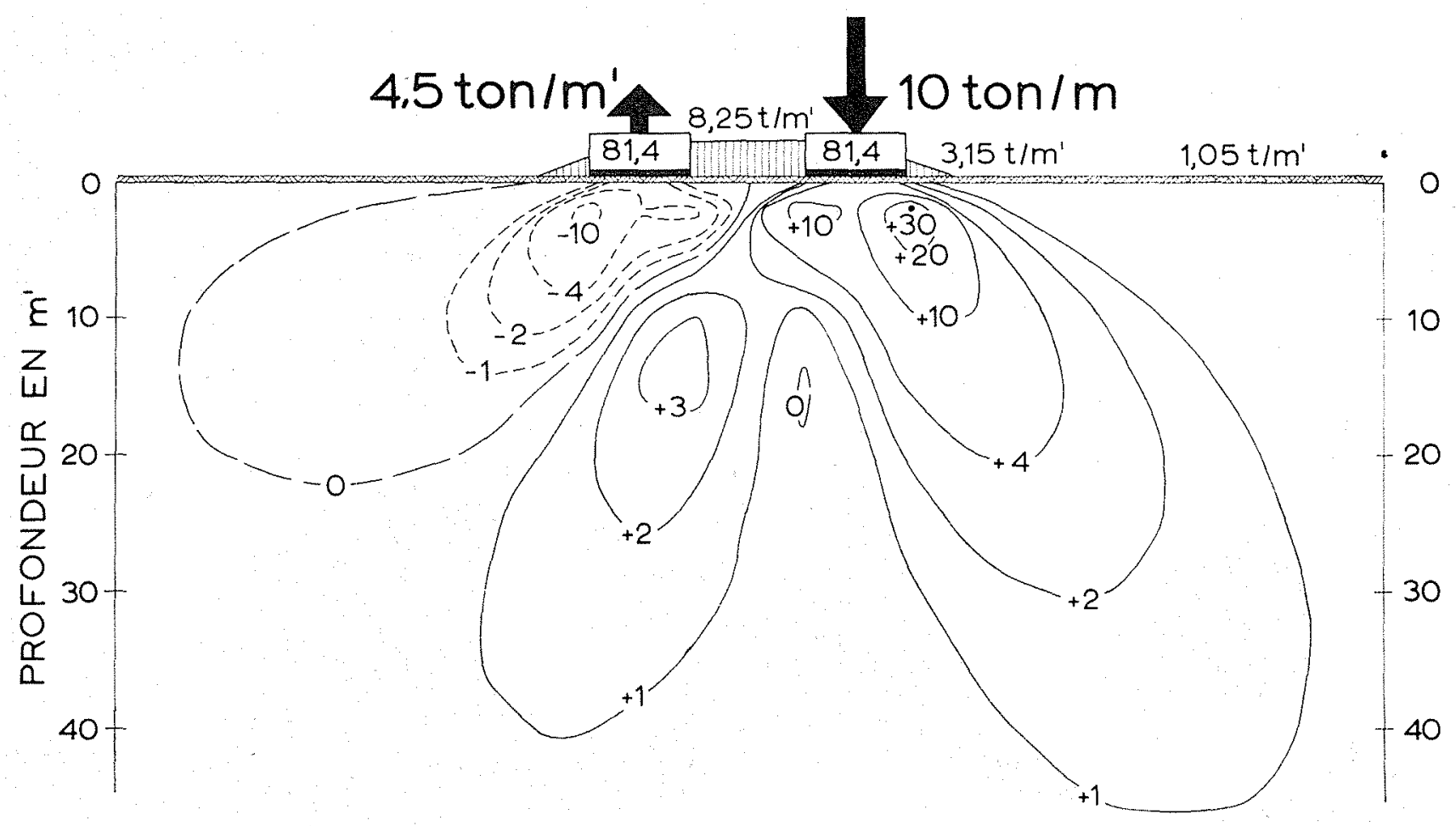

FIg. 21

Charges dynamiques.

Pressions hydrostatiques en décimètres de colonne d'eau.

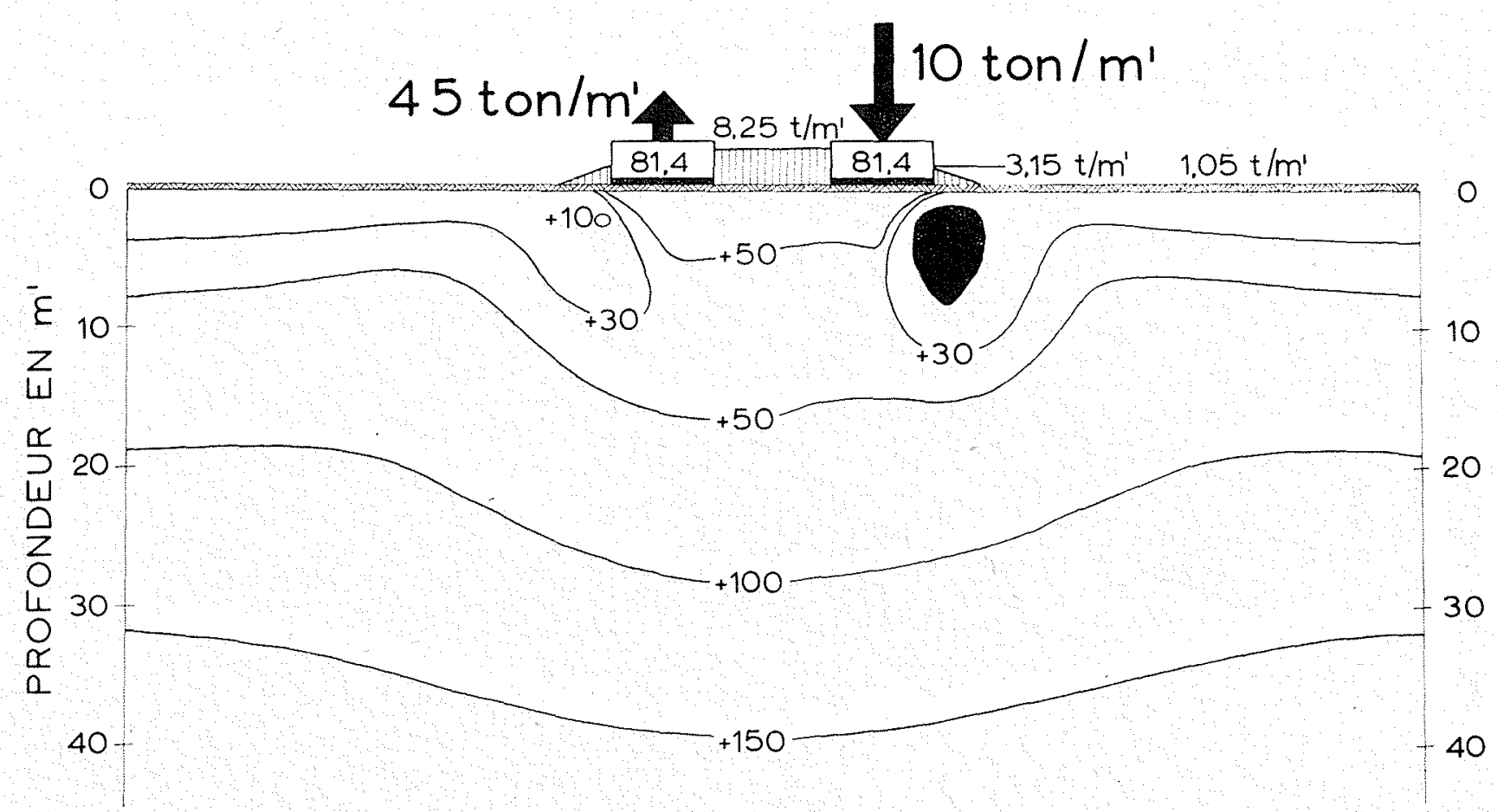

FIG. 22

Difference entre pressions hydrostatiques admissibles et véridiques (en $\mathrm{dm}$ ). $\varphi=20^{\circ} \quad h=0,70 \quad$ volume interstitiel $=45 \%$. 


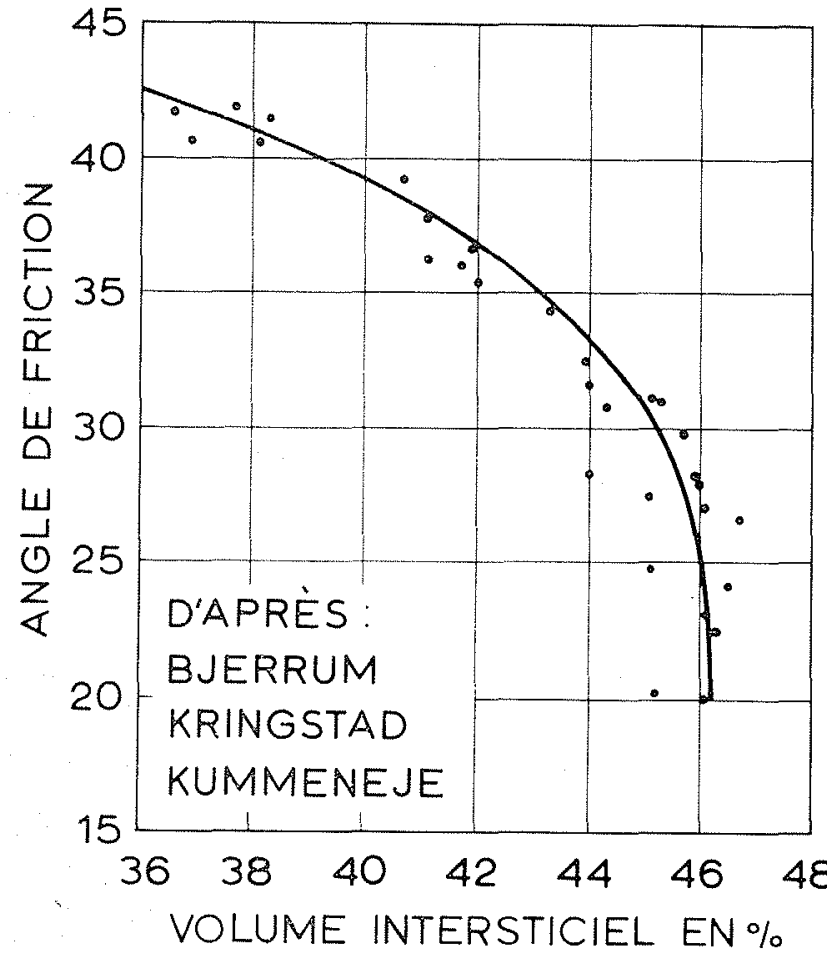

Les surpressions hydrostatiques qui résulteront de ces chargements rapides peuvent hien, en principe, être calculées. En plus des susdites fatigues de cisaillement, on devra, cela va sans dire, tenir compte des fatigues normales. La tigure 21 donne le résultat d'un tel calcul. Il s'agit ici du pylône d'un téléférique blondin porteur de bennes automotrices. Les surpressions hydrostatiques qu'il y a lieu d'attendre dans du sable de tassement lầche, comme conséquence de ees chargements à croissance relativement rapide, sont indiqués sur cette figure comme courbes de pression égale exprimée en décimètres d'eau. Lors de ces chargements relativement faibles, il peut done encore se produire des pressions hydrostatiques importantes.

Pour examiner si ces surpressions hydrostatiques constituent un inconvénient, on peut les comparer aux pressions hydrostatiques où il ne se produirait justement pas d'affaissement. La figure 22 indique le domaine où les pressions

8 FIG. 23

Angle de friction $\varphi$

en fonction du degré de tassement du sable.

FIG. 24

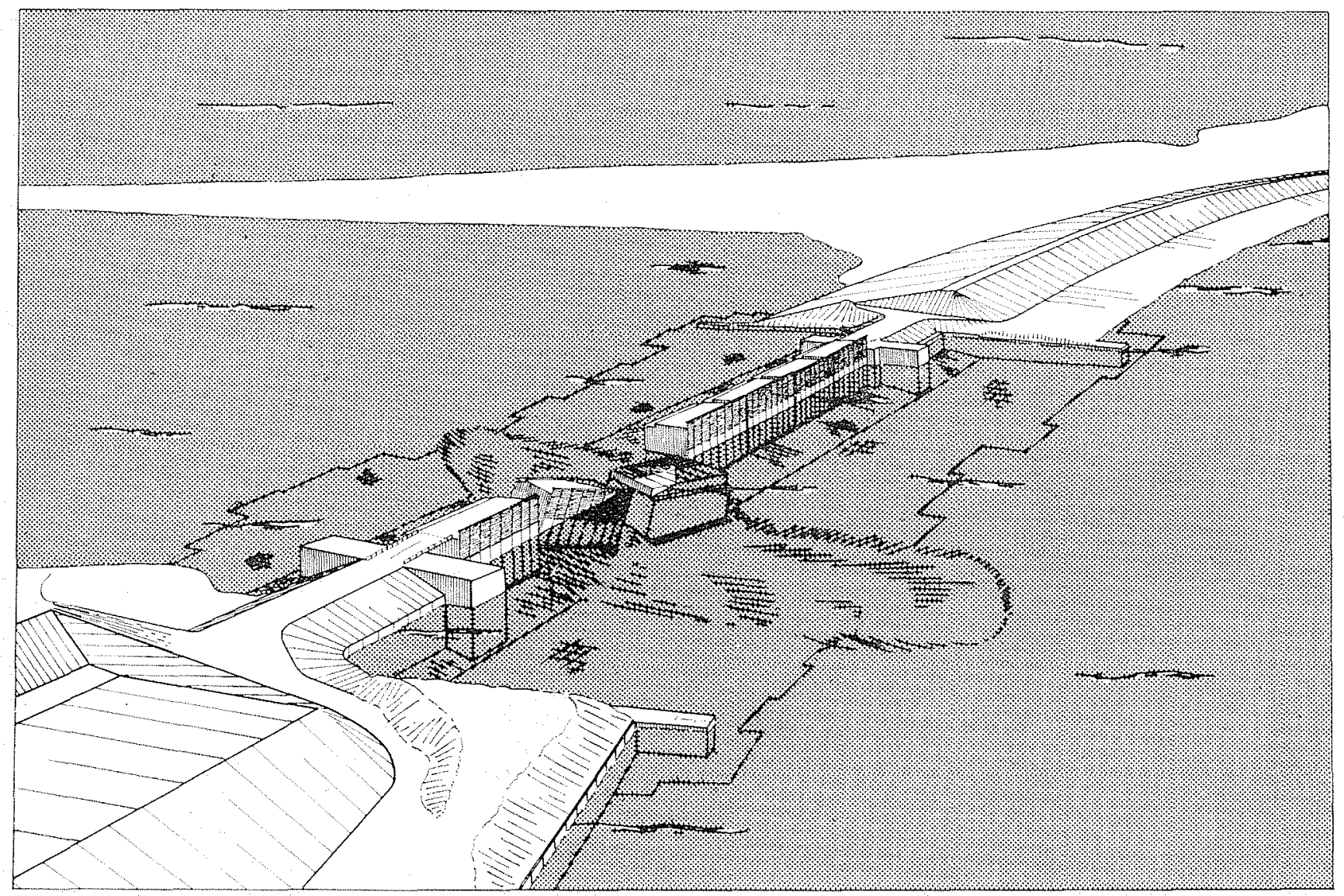




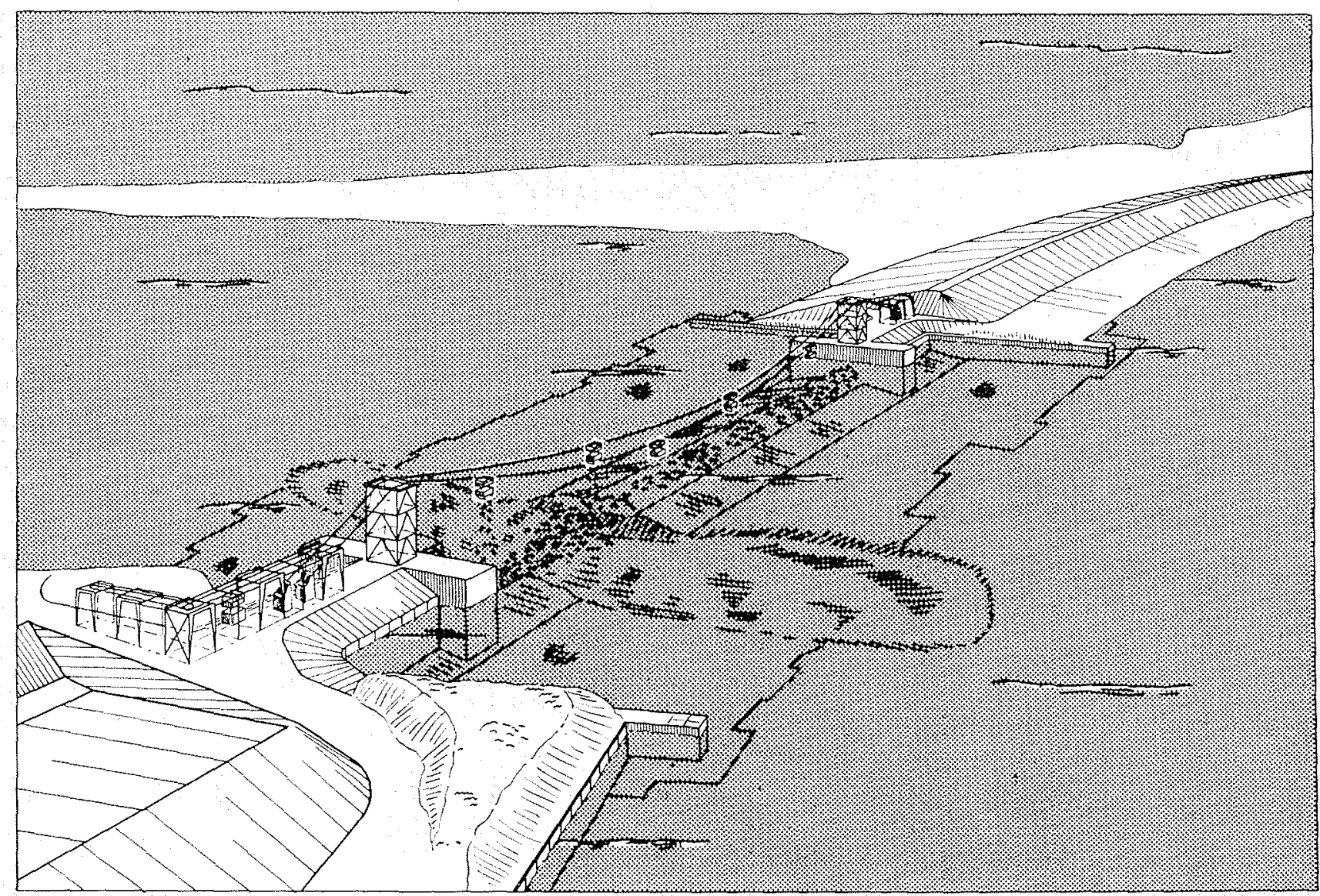

FIG. 25

hydrostatiques qui surviennent sont plus élevées que celles qui sont admissibles. On voit que les pressions hydrostatiques qui sont une conséquence de la propriété de variation de structure du sol peuvent être déjà dangereuses, même en cas de petits chargements.

Pour calculer les pressions hydrostatiques qui sont admissibles, on part de l'hypothèse d'un angle de friction (仓) de $20^{\circ}$. Ce faible angle de friction a une raison intentionnelle. Des essais ont montré en effet que les valeurs o du sable dépendent de la densité de tassement.

Cela est exprimé sur la figure 23. C'est surtout lorsque le tassement est très lâche, et par conséquent les espaces intersticiels sont larges, qu'on voit la valeur diminuer jusqu'à une valeur très faible.

Le sable à tassement lâche possède donc deux propriétés dangereuses. En premier lieu, des surpressions hydrostatiques, qui réduisent la résistance à la fatigue de cisaillement peuvent survenir; en second lieu, le sable à tassement lâche a un petit angle de friction.

Ces deux propriétés font que, dans du sable à tassement lâche, il peut surgir facilement une perturbation locale de l'équilibre. Cette perturbation de l'équilibre peut produire ailleurs de nouvelles pressions hydrostatiques et une espèce de réaction en chaîne peut en résulter; elle peut même acquérir une vaste ampleur. On nomme ce phénomène un fluage.

Si un tel fluage se produisait pendant une fermeture avec des caissons, cela pourrait avoir de graves conséquences. C'est ce que la figure 24 montre. Les caissons patinent ou se renversent. Le courant peut de nouveau pénétrer par l'ouverture et il va aggraver les ravages. Restaurer un tel désastre exigerait des mois, si ce n'est des années.

Si l'on procède à une fermeture progressive, en larguant à l'aide d'un téléférique les matériaux de remblai, nécessaires au seuil, on peut toujours prendre soin, par exemple par une fondation sur pieux, que la stabilité des pylònes du téléférique reste assurée en tout temps.

Si le même fluage se produisait, il n'y aurait vien d'autre à faire que de continuer à larguer les matériaux. On concentrera alors la pleine capacité du téléférique sur le point avarié, ce qui rendra possible un rapide rétablissement. 


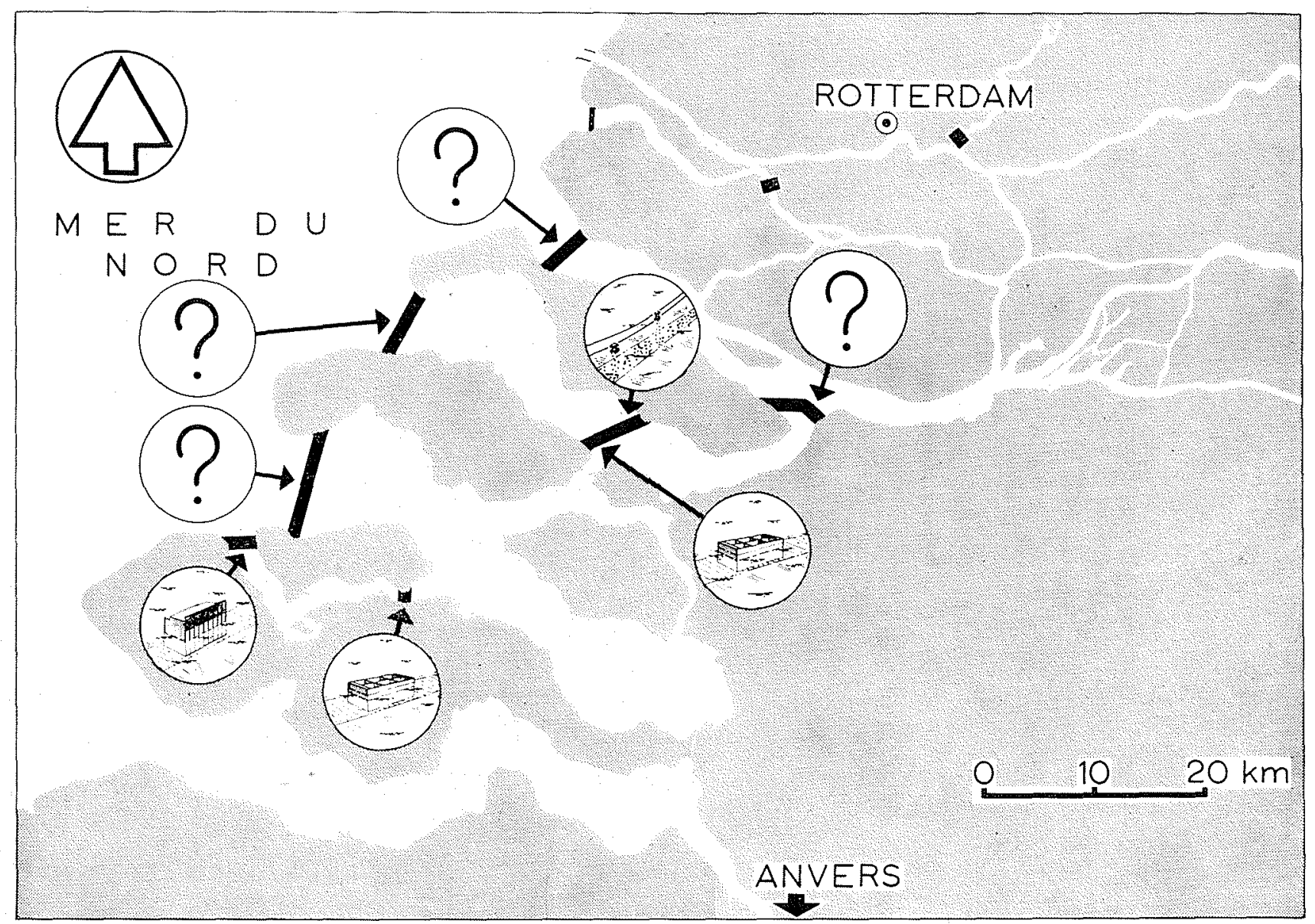

Fig. 26

Ce rétablissement plus facile après un éventuel fluage est la raison qui fait qu'en négligeant la question du prix, on préfère dans certains cas une fermeture progressive des passes moins profondes plutôt qu'une fermeture avec des caissons évidés.

Peut-être attendra-t-on que je termine en disant de quelle manière nous fermerons finalement les larges estuaires, si difficiles à barrer? $\mathrm{Si}$ c'est le cas, je vais vous décevoir (fig. 26). Vous avez certainement deviné que, pour être plus clair, j'ai présenté les divers problèmes qui jouent un rôle dans le choix de la méthode de fermeture, d'une façon un peu plus simple qu'ils ne le sont en réalité. De plus, un grand nombre de données qui servent de base font encore défaut. J'ai peut-être bien rendu plausible à l'aide d'un sondage et d'un forage, qu'il pourrait être question de sable à tassement lâche, mais je n'ai pas encore pu vous dire quelles sont exactement les proportions des espaces intersticiels de ce sable.
Je n'ai pas non plus encore parlé de nombreux problèmes essentiels, parce qu'on traitera ces sujets lors des exposés suivants. On verra alors qu'ils sont loin d'avoir été tous résolus.

Enfin nous restons encore sur notre position que, pour la fermeture des larges estuaires, nous ne voulons appliquer aucune méthode pour laquelle nous n'avons pas encore acquis d'expérience. Nous avions déjà de l'expérience avec divers genres de fermetures par caissons. L'année prochaine, nous espérons en acquérir avec la fermeture progressive par remblayage au moyen d'un téléférique. A ce moment-là, tel est notre espoir, les diverses études aussi seront plus avancées que maintenant. La décision qui concerne la méthode à suivre pour la fermeture des larges estuaires sera donc prise au plus tôt dans un an environ.

Cependant j'espère bien que nous pourrons tracer à grands traits et clairement quelles considérations il faut retenir sur ce sujet. 


\section{I S C U S I O N}

président : M. DE RouviLLE

M. le Président remercie M. Bischoff Van Heemskerck de son exposé, qui traite d'un problème très délicat. Sur l'une des figures, il y avait quatre points d'interrogation et quatre points de certitude. On attendra, avec patience, la résolution de es points d'interrogation.

A une question de $M$. le Président sur la possibilité de choisir la place de la fermeture, M. Brschorf Van Hezaskenck indique que le sondage et le forage montrés sont représentatifs des estuaires. Du fait de notre connaissance accumulée du sol, on ne s'attend pas à trouver des endroits qui présentent des conditions plus favorables. De toute façon, on ne peut pas compter sur des stratifications rocheuses.

M. Mrche désirerait obtenir quelques informations complémentaires sur le mode de réalisation et sur la stabilité à titre provisoire de la fermeture du Veerse Gat exécutée récemment au moyen des caissons de béton armé munis de vannes. Il présume que ces dernières ont été placées à l'amont et que lorsqu'elles ont été fermées, le bassin intérieur était sensiblement au niveau moyen de la mer. De cette façon, les caissons disposaient d'une charge d'eau supplémentaire à marée haute, ce qui était favorable. Toutefois, la prédominance des poids n'apparaît pas très importante pour ces caissons soumis à des efforts alternés dus à la marée et à la houle. C'est sans doute par suite du marnage relativement faible que la stabilité a pu rester assurée.

En ce qui concerne, à titre comparatif, la fermeture de l'estuaire de la Rance où les marées sont très fortes, les études préalables avaient montré qu'on ne pouvait pas assurer de cette manière la stabilité avec des caissons de dimension raisonnable, sauf à les remplir complètement de sable. Or, cette opération ne pouvait être réalisée dans l'intervalle d'une marée lorsqu'il y a un certain nombre de caissons et l'on a dû procéder autrement.

Il semble done qu'en Hollande, par suite des conditions locales, cet aspect de la question perd de son acuité et que les préoccupations principales restent celles relatives à la stabilité du matériau sableux constituant la fondation des caissons.

M. Bischoff Van Heenskenck dit qu'en effet le problème de la stabilité des caissons est facilement résolu car la marée est faible $(1,60 \mathrm{~m}$ environ) et l'on ajoute par remblayage des charges lourdes sur le plafond des. caissons, de façon à ne pas obturer les pertuis. Ce travail fut réalisé pendant l'étale chaque fois qu'un caisson avait. été coulé.

Dans le cas du Veerse Gat, on avait affaire à sept grands caissons qui furent coulés - à une exception près -a au cours de sept jours consécutifs.

M. Marc Henry a été très intéressé par l'exposé, et notamment par l'évocation du fluage du sable.

Ce fluage, bien connu en mécanique des sols, constitue une propriété de certains sables à granulométrie très uniforme. Lorsque, étant saturés, ces sables sont soumis à des cisaillements, leur comportement dépend de leur degré de tassement, qu'on peut caractériser par leur densité sèche:

- quand la densité sèche est supérieure à une densité sèche critique, qui est de l'ordre de 1,5 à 1,6 , la rupture du sable comprimé s'accompagne d'une augmen. tation de volume, laquelle ne peut se faire que moyennant un apport d'eau interstitielle;

- inversement, quand la densité sèche est inférieure à la densité sèche critique, la rupture s'accompagne d'une diminution du volume, laquelle ne peut se faire que moyennant expulsion d'eau interstitielle. Dans certains cas, cette rupture est brutale, et l'eau interstitielle en excédent se trouve brusquement libérée, en sorte que les grains cessent d'être en contact. La résistance au cisaillement est momentanément annulée, le sable se trouvant liquéfié;

- or, lorsque ces sables sont mis en place par un fluide en état d'agitation (air, cours d'eau, mer), l'expérience montre que leur densité sèche est nettement inférieure à la densité sèche critique : la densité sèche varie couramment entre 1,2 et 1,4 , la valeur moyenne de 1,3 correspondant à un arrangement à $50 \%$ de vides;

- on s'explique ainsi que le chargement de sable de mer saturé conduise à des tassements très importants, susceptibles de se produjre brutalement: c'est le fluage, dont a parlé le conférencier.

Pour éviter ou réduire le fluage, on peut envisager, en théorie tout au moins, de serrer le sable préalablement à son chargement :

- ce serrage peut se faire par pervibration, ou par fongage de pieux;

- on peut l'obtenir également en établissant dans ce sable des puits filtrants, et en pompant dans ces puits. On établit ainsi dans le sable des pressions de courant qui équivalent à un préchargement.

L'expérience montrant que, sur le tracé d'une digue de fermeture, les caractéristiques du sable sont partout médiocres, ce qui ne permet pas d'implanter la passe de coupure dans une région où le sable serait plus consistant, M. Marc Henry demande s'il ne serait pas possible de réaliser, par une des méthodes qu'il vient de décrire, une consolidation préalable du sable, à l'emplacement choisi pour la passe de coupure.

M, Brschoff VAN Heemsinenck répond qu'il est possible d'améliorer le sable par un vibrateur dans une région de 3 ou $4 \mathrm{~m}$; le faire sur $7 \mathrm{~km}$ revient très cher. La raison pour laquelle on cherche d'autres méthodes, c'est de localiser et de limiter les conséquences d'un fluage, comme cela a été rendu clair dans la flgure donnant un croquis pronostiquant l'allure d'un tel contrecoup dans les deux cas de fermeture par caissons et remblayage progressif.

Sur des questions de M. le Président, M. Bischorf $V_{A N}$ HeEMsiencr indique que l'on n'a fait que des hypothèses sur l'injection de ciment et que le froid est une méthode aussi très coûtense.

M. Robert rappelle que M. Miche a signalé qu'il y avait là une question de la fondation de caissons sur une plate-forme de sable. Les ingénieurs néerlandais savent sûrement que $M$. CaQuot a donné une formule de calcul de la contrainte admissible sur un tel sol. On a pu généraliser cetfe formule en tenant compte, d'une part, des composantes horizontales de la contrainte d'appui, d'autre part, de l'inclinaison du talus créé par les 
affouillements. Ces paramètres, le calcul théorique le montre, réduisent considérablement, en certains cas, la contrainte admissible sur la plate-forme d'appui. En pratique, d'ailleurs, la difficulté est bien celle de connaître les caractéristiques du sable de fondation, angle $\varphi$ et densite apparente, en relation avec les risques de se trouver dans une zone susceptible de se liquéfier.

M. Bischorf VAN Heemsienck eroit bien connaitre les calculs de M. Cagvot, mais il lui semble que les calculs en question font suite aux calculs des pressions hydrostatiques. On n'a voulu que donner un exemple d'un tel calcul.

M. Stenling, au sujet de l'observation faite par M. Mrche, fait remarquer qu'il n'y a pas lieu de lester tous les caissons simultanément. Après la pose de chaque caisson, on dispose d'un certain délai - 24 heures au moins - afin de lui assurer une stabilité sulfisante pour résister aux sollicitations auxquelles il sera soumis au moment de la fermeture des vannes, qui n'a lieu qu’après la pose du demier caisson.

M. Miche se demandait également si les ingénieurs néerlandais avaient envisagé de couler simultanément les sept caissons du Veerse Gat.

M. Bischoff Van Heemskeres indique qu'on ne peut couler que quatre caissons par jour au plus. Une fois que la totalité des caissons est coulée, ce sont les vannes que l'on ferme simultanćment.

M. Mrene constate que lorsqu'on ferme les vannes simultanément, les caissons encaissent la différence de pression d'ean due à la maxée. $O r$, comme il faut faire flotter les caissons, ils doivent être relativement légers, d'où l'intérêt de la surcharge avant la fermeture des vannes signalée précédemment par $M$. Bischoff VAN Henaskenck. Si cette surcharge ne peut etre mise en place dans l'intervalle d'une demi-marée, il faut donc charger les caissons, vamnes ouvertes et au-dessus des pertuis de passage, ce qui apparait être la solution utilisée; elle serait alors applicable, même s'il y avait plus de sept caissons

M. Bischoff VAN HeEMsnerick répond affirmativement mais indique qu'il reste le problème de la stabilité des caissons, car l'attaque des vagues peut être considérable au cours d'un laps de temps d'un mois environ qui est nécessaire pour conler les 70 caissons.

M. le Président pense qu'il y a lieu de distinguer deux périodes : celle où l'on ferme la plus grande partie de la digue, puis celle de la pose des caissons de fermeture pour laquelle on profitera d'une période calme. De quel ordre de grandeur est la largeur du barrage qui reste critique pour une fermeture?

M. Bischoff Van Hremskenck répond que cela dépend du volume de marée. Il y a des bancs de sable et des chenaux; on peut construire au-dessus des bancs, mais le danger commence quand on va construire dans les chenaux. Il faut le faire rapidement, le temps est l'élément essentiel.

M. le Président pense que les auditeurs ont maintenant une idée plus précise de la difficulté du problème.

M. le Président remercie à nouveau le conférencier dont le rapport était très intéressant.

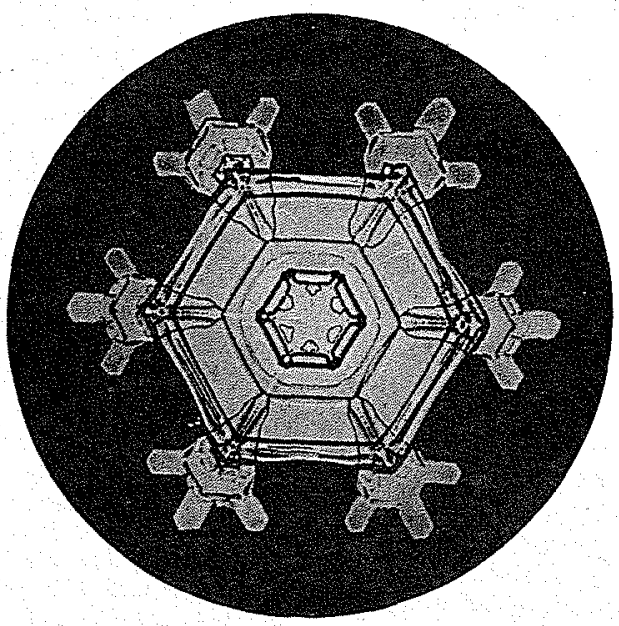

